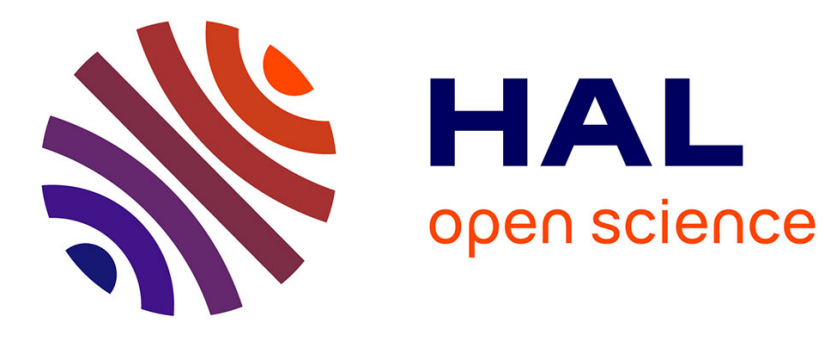

\title{
Characterization of road microtexture by means of image analysis
}

Anis Ben Slimane, Madji Khoudeir, Jacques Brochard, Minh Tan Do

\section{To cite this version:}

Anis Ben Slimane, Madji Khoudeir, Jacques Brochard, Minh Tan Do. Characterization of road microtexture by means of image analysis. 10th International Conference Metrology and Properties of Engineering Surfaces, Jul 2005, Saint-Etienne, France. 8p, ill., schémas, graphique. hal-00851279

\section{HAL Id: hal-00851279 https://hal.science/hal-00851279}

Submitted on 13 Aug 2013

HAL is a multi-disciplinary open access archive for the deposit and dissemination of scientific research documents, whether they are published or not. The documents may come from teaching and research institutions in France or abroad, or from public or private research centers.
L'archive ouverte pluridisciplinaire $\mathbf{H A L}$, est destinée au dépôt et à la diffusion de documents scientifiques de niveau recherche, publiés ou non, émanant des établissements d'enseignement et de recherche français ou étrangers, des laboratoires publics ou privés. 


\title{
CHARACTERIZATION OF ROAD MICROTEXTURE BY MEANS OF IMAGE ANALYSIS
}

\author{
Anis Ben Slimane \\ Laboratoire Signal, Image et Communications (SIC) \\ Bd Marie et Pierre Curie - BP 30179, 86962 Futuroscope Chasseneuil Cedex \\ Phone : +33 5494965 85, Fax: +33 549496567 \\ Email: slimane@sic.sp2mi.univ-poitiers.fr \\ Majdi Khoudeir \\ Laboratoire Signal, Image et Communications (SIC) \\ Bd Marie et Pierre Curie - BP 30179, 86962 Futuroscope Chasseneuil Cedex \\ Phone: +33 5494965 85, Fax: +33 549496567 \\ Email: khoudeir@sic.sp2mi.univ-poitiers.fr \\ Jacques Brochard \\ Laboratoire Signal, Image et Communications (SIC) \\ Bd Marie et Pierre Curie - BP 30179, 86962 Futuroscope Chasseneuil Cedex \\ Phone : +33 5494965 85, Fax: +33 549496567 \\ Email :Brochard@sic.sp2mi.univ-poitiers.fr \\ Minh-Tan Do \\ LCPC \\ Route de Bouaye - BP 4129, 44341 Bouguenais - France \\ Phone : +33 2408457 95, Fax: +33240845992 \\ Email : minh-tan.do@lcpc.fr
}

\begin{abstract}
:
Road-surface microtexture (sub-millimeter scale) is essential for pavement skid-resistance. However, its measurement is only possible in laboratory on cores taken from trafficked roads, and is time consuming. For an efficient road monitoring, it is necessary to develop faster methods and usable on-site.

Collaboration has been developed for two years between LCPC and the laboratory Signal, Image and Communication (SIC) to develop a measurement and characterization method for road microtexture based on image analysis.

This paper deals with tow complementary works:

- $\quad$ The measurement of road microtexture. Research is focused on the image measurement and extraction of roughness information from images. The prototype using a high-resolution camera is described. The procedure separating relief- from aspect-information using a photometric model for the surface is given. Image-based relief variation is compared to relief variation obtained through laser sensor.

- The characterization of road microtexture. This characterization, obtained through a geometrical and frequential analysis of images, leads to descriptors related to the shape and the density of surface asperities.

Experimental campaigns were carried out to validate the feasibility of measuring on-site images and to correlate surface descriptors to friction. Results are presented and discussed. Perspective for future works is given.
\end{abstract}

Key words: image analysis, photometric stereovision, roughness criteria, road surface. 


\section{Introduction}

Important development of road network in France has led to the development of tools for road condition diagnosis. Regarding road surface texture, operating tools are based on profile measurements [1]. Using laser sensors, surface irregularities from centimeter- to millimeter scales called " macrotexture », can be currently measured. There exists no onboard equipment for the measurement of the sub-millimeter scale called "microtexture ", which is also relevant for the assessment of pavement skid-resistance.

Regarding the fine dimension of road microtexture, the main drawback of laser-based systems is the acquisition time, which is too long for measurements on trafficked roads. Collaboration has been then developed between LCPC and SIC laboratory (Signals, Images and Communications) to study the feasibility of measuring road microtexture by means of image-based systems.

The first step of this collaboration is the development of a transportable device for staticcondition measurement and a microtexture description method. Works are reported in this paper.

\section{Road texture and pavement skid-resistance}

Road surface irregularities are divided into two scales [2]:

- the macrotexture corresponding to surface irregularities which horizontal dimensions are between $0.5 \mathrm{~mm}$ and $50 \mathrm{~mm}$, and vertical dimensions are between $0.1 \mathrm{~mm}$ and 20 $\mathrm{mm}$;

- the microtexture corresponding to surface irregularities which horizontal dimensions are less than $0.5 \mathrm{~mm}$, and vertical dimensions are between $0.001 \mathrm{~mm}$ and $0.5 \mathrm{~mm}$.

It is admitted that the macrotexture is required for water drainage at tire/road interface, and that the microtexture is important for friction force generation.

\section{Research methodology}

Road surfaces can be considered as $3 \mathrm{D}$ textured images in which micro-asperities existing on aggregate surfaces are the microtexture, and aggregate contours are the macrotexture. The difficulty is to extract from image information those related to surface relief. Actually, roughness characterizations based on image analysis assume generally that grey-level variations are representative of relief variations [3-5]. This assumption might be valid for uniformly colored surfaces ; it is questionable for non uniformly colored surfaces. In the last case, grey-level variations are due to both relief and color. It is then necessary, before starting the characterization, to separate in the same image relief- from color information. Filtering approaches based on wavelets were developed for this purpose [6,7]. Nevertheless, relief smoothing can be induced and these approaches can be inefficient in case where spatial frequencies of relief- and color information are the same.

In this study, a photometric stereovision method is proposed. The color/relief separation is done from three images taken at three different lighting conditions and from a photometric model of the surface.

The relevance of the proposed method is assessed by means of analyses of surfaces representative of French roads. Correlation between image descriptors and friction values is done. In the following paragraph, image descriptors are presented.

\section{Presentation of « image » roughness criteria}

\subsection{Geometrical criterion: Analysis of gradient distribution}

The gradient operator is a suitable means to observe local surface variations. The slope in each surface point reveals height variation representative of the microtexture. Generally, for 
rough textured surface, slope distribution is a zero-mean Laplacian. The Laplacian standard deviation represents indirectly the surface roughness: the more the standard deviation is high, the more the surface is rough. The standard deviation of gradient distribution is then relevant for assessing surface roughness.

Noting $p=\partial z / \partial x$ and $q=\partial z / \partial y$ and considering $G=\{p, q\}$ the slope population of the surface $z(x, y)$, the gradient criteria is calculated as :

$$
C=\sqrt{\frac{1}{N} \sum_{n=1}^{N}\left(G_{n}-\bar{G}\right)^{2}}
$$

where $N$ is the individual number of $G$ et $\bar{G}$ is its average given by: $\bar{G}=\frac{1}{N} \sum_{n=1}^{N} G_{n}$

Since the surface shape is plane, $\bar{G}$ should be equal to zero. So the criterion becomes:

$$
C=\sqrt{\frac{1}{N} \sum_{n=1}^{N}\left(G_{n}\right)^{2}}
$$

Discontinuities between coarse aggregates induce high gradient values. These values affect the calculated criteria, but they are not representative of the microtexture. In consequence, they must be discarded.

\subsection{Frequency criterion: Power Spectrum Density}

Surface cartographies comprise low-frequency information related to surface global structure (coarse aggregate distribution), and high-frequency information related to surface local structure (micro asperities to be characterized). A simple idea is to study the spectral distribution of relief energy, in other words the power spectrum density (PSD), and precisely the relationship between low- and high frequencies. PSD is normalized by putting the maximum equal to 1 , in order to compare surfaces. The criterion is then the PSD standard deviation. Actually, PSD represents a band pass of a function and reflects the frequency distribution in this band. The more this band pass is large, the more the contribution of high frequencies is important. The standard deviation can represent the band pass width and will be useful since it is related to the aggregate density.

The PSD is defined by: $\operatorname{PSD}^{2}(\mu, v)=|\operatorname{TF}(z(x, y))|=|Z(\mu, v)|$

where $Z(\mu, v)$ is the Fourier transform of $z(x, y)$ and $\mu, v$ frequencies corresponding respectively to $x$ and $y$ axis.

\subsection{Mixed criterion}

It is necessary to combine frequential and geometrical characteristics since both asperity density and their angularity define surface roughness. It can be noted that the density and angularity effects are multiplicative and not additive. Actually, for the same aggregate angularity, surfaces will be (n) times rougher if they are covered by (n) times more of aggregates.

Roughness analysis based on images using the above criteria is only possible in case where grey-level variations represent local surface-relief variations. This restrictive assumption requires no surface-color or aspect variation. It implies specific study on photometric behaviour of road surfaces. Another way is to extract relief information from images by means of photometric stereovision technique. 


\section{Relief extraction by photometric stereovision}

\subsection{Images}

Specimens are cores taken from various wearing courses. The skid-resistance of each core is characterized by friction coefficient measured by a portable device called "Skid Resistance Tester » (SRT) [1]. Specimen surfaces were photographed using CCD camera under controlled lighting conditions. Since microtexture is concerned, finest resolution was requested; it was still higher than 50 microns.

\subsection{Link between textured surface image and relief}

Since surfaces are not specular, Lambertian model was used to characterize their photometric behaviour and to set the link between grey level, color information and relief. Let us consider a rough textured surface composed of Lambertian micro-facets. The surface is lighted under incidence angle $\theta_{i}$ and observed by camera mounted perpendicularly to the surface plane ( $x, y)$ (Fig. 1). Definitions of the angles shown in the figure 1 are the following:

$\theta_{i}$ : incidence angle related to the surface ;

$\varphi$ : incidence angle related to the facets ;

$\alpha$ : angle between the surface normal and the facet normal.

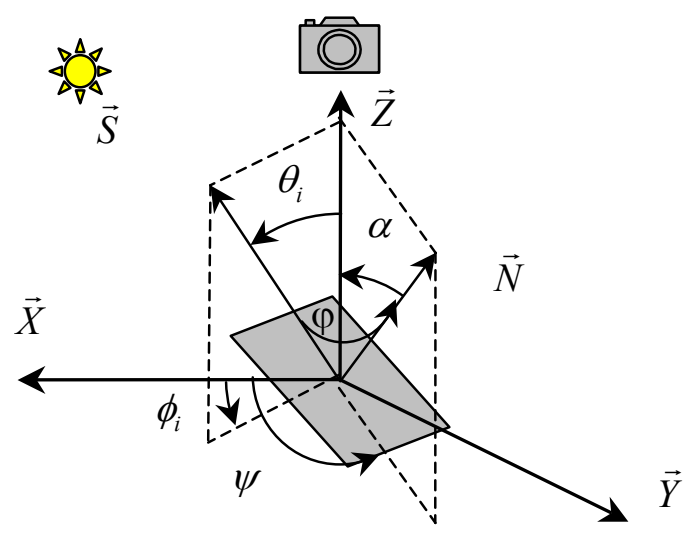

Fig. 1: Configuration for view shot.

In case of lambertian surface, the image intensity $\mathrm{I}(\mathrm{x}, \mathrm{y})$ represents the energy received by the CCD sensor. It is expressed by the following relation:

$I(x, y)=\frac{L(x, y)}{r^{2}} \cos \varphi(x, y)$

where

$I(x, y)$ : Image intensity;

$L(x, y)$ : coefficient representative of surface colorimetric properties;

$\varphi(x, y)$ : incidence angle related to the facet ;

$r$ : distance between the lighting source and the facet.

The distance will be assumed to be constant since relief variations are negligible compared with the distance surface/lighting source.

We try then to express the angle $\varphi(x, y)$ as a function of cartesian coordinates $(\mathrm{x}, \mathrm{y}, \mathrm{z})$ of the current point. The following vectors are defined: 
$\overrightarrow{\mathrm{Z}}=\left[\begin{array}{lll}0 & 0 & 1\end{array}\right]^{\mathrm{T}}$, vector normal to the surface plane;

$\vec{S}=\left[\sin \theta_{i} \cos \phi_{i} \sin \theta i \sin \phi_{i} \cos \theta\right]^{T}$, the unit vector of the light source direction, $\phi_{i}$ is the angle between $\overrightarrow{\mathrm{x}}$ axis and the projection of the lighting source vector $\vec{S}$ in the plane $(\overrightarrow{\mathrm{x}}, \overrightarrow{\mathrm{y}})$.

$\vec{N}=\left[\begin{array}{lll}a & b & c\end{array}\right]=[\sin \alpha \cos \psi \sin \alpha \sin \psi \cos \alpha]$, the normal to the facet.

Let us consider a point $M$ from the surface. The relief in the neighbourhood of this point can be considered as a plane defined by the following equation:

$\mathrm{P}: \mathrm{ax}+\mathrm{by}+\mathrm{cz}=0$

So, we obtain :

$\cos \varphi=\vec{N} \vec{S}=\left[\begin{array}{lll}\sin \alpha \cos \psi & \sin \alpha \sin \psi & \cos \alpha\end{array}\right]\left[\begin{array}{lll}\sin \theta_{i} \cos \phi_{i} & \sin \theta_{i} \sin \phi_{i} & \cos \theta_{i}\end{array}\right]^{T}$

So : $\cos \varphi=\cos \alpha\left(\tan \alpha \cos \psi \sin \theta_{i} \cos \phi_{i}+\tan \alpha \sin \psi \sin \theta_{i} \sin \phi_{i}+\cos \theta_{i}\right)$

From the equation $\mathrm{P}: \mathrm{ax}+\mathrm{by}+\mathrm{cz}=0$, we obtain:

$$
z(x, y)=-\frac{a}{c} x-\frac{b}{c} y \Rightarrow\left\{\begin{array}{l}
\frac{\partial z}{\partial x}=-\frac{a}{c} \\
\frac{\partial z}{\partial y}=-\frac{b}{c}
\end{array}\right.
$$

Combining the equation of $\overrightarrow{\mathrm{N}}$ with equation (9), we obtain:

$\left\{\begin{array}{l}\frac{\partial z}{\partial x}=-\frac{a}{c}=-\tan \alpha \cos \psi \\ \frac{\partial z}{\partial y}=-\frac{b}{c}=-\tan \alpha \sin \psi\end{array}\right.$

We deduce then $\cos \alpha$ as: $\cos \alpha=1 / \sqrt{1+(\partial z / \partial x)^{2}+(\partial z / \partial y)^{2}}$

Finally, from equations (5), (8), (10) et (11), we obtain:

$I(x, y)=\frac{L(x, y)}{r^{2}} \frac{\cos \theta_{i}-\frac{\partial z}{\partial x} \sin \theta_{i} \cos \phi_{i}-\frac{\partial z}{\partial y} \sin \theta_{i} \sin \phi_{i}}{\sqrt{1+(\partial z / \partial x)^{2}+(\partial z / \partial y)^{2}}}$

The above expression enables us to differentiate different information included in the image grey level.

\subsection{Method to extract information related to relief}

Equation (12) is employed to extract the relief information from the image. This equation comprises three unknowns, two of which are related to relief variations $(\partial z / \partial x$ et $\partial z / \partial y)$ and 
one to surface colorimetric properties $\mathrm{L}(\mathrm{x}, \mathrm{y})$. We have to solve a system with three equations and three unknowns. To produce the equations, three images are recorded. They correspond to the same surface lighted under three incidence angles. The incidence angles $(\theta)$ are low to prevent shadows in the images.

Let $I_{1}, I_{2}$ et $I_{3}$ be the images shot successively under the following lighting conditions : $\left(\theta_{1}=\theta\right.$ and $\left.\phi_{1}=0\right),\left(\theta_{2}=\theta\right.$ and $\left.\phi_{2}=2 \pi / 3\right)$ et $\left(\theta_{3}=\theta\right.$ and $\left.\phi_{3}=-2 \pi / 3\right)$.

The 3-equation system is the following :

$$
\left\{\begin{array}{l}
I_{1}(x, y)=L(x, y) \frac{\cos \theta-\frac{\partial z}{\partial x} \sin \theta}{\sqrt{1+(\partial z / \partial x)^{2}+(\partial z / \partial y)^{2}}} \\
I_{2}(x, y)=L(x, y) \frac{\cos \theta+\frac{1}{2} \frac{\partial z}{\partial x} \sin \theta-\frac{\sqrt{3}}{2} \frac{\partial z}{\partial y} \sin \theta}{\sqrt{1+(\partial z / \partial x)^{2}+(\partial z / \partial y)^{2}}} \\
I_{3}(x, y)=L(x, y) \frac{\cos \theta+\frac{1}{2} \frac{\partial z}{\partial x} \sin \theta+\frac{\sqrt{3}}{2} \frac{\partial z}{\partial y} \sin \theta}{\sqrt{1+(\partial z / \partial x)^{2}+(\partial z / \partial y)^{2}}}
\end{array}\right.
$$

Its resolution gives:

$$
\left\{\begin{array}{l}
\frac{\partial z}{\partial x}=-\frac{\left(2 I_{1}-I_{2}-I_{3}\right) \cos \theta}{\left(I_{1}+I_{2}+I_{3}\right) \sin \theta} \\
\frac{\partial z}{\partial y}=-\frac{\sqrt{3}\left(-I_{2}+I_{3}\right) \cos \theta}{\left(I_{1}+I_{2}+I_{3}\right) \sin \theta}
\end{array}\right.
$$

\subsection{Experimental results}

Examples of images are shown in the figure 2. The camera/surface distance is $1 \mathrm{~m}$. Lighting conditions are respectively $\left(\theta_{1}\right.$ and $\left.\phi_{1}\right),\left(\theta_{2}\right.$ and $\left.\phi_{2}\right)$ and $\left(\theta_{3}\right.$ and $\left.\phi_{3}\right)$. The angle $\theta \mathrm{i}$ was low to prevent shadows.

In the figure 2, the image on the left is one of those captured by the camera. On the right, the image represents relief variations, expressed as grey level (the " white » level corresponds to maximum height). We can see that the correspondence between the surface photography and the extracted relief is good. 

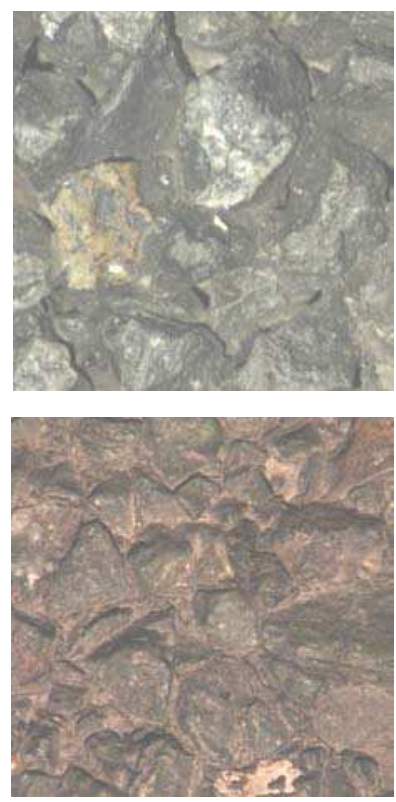

One of the three acquired images
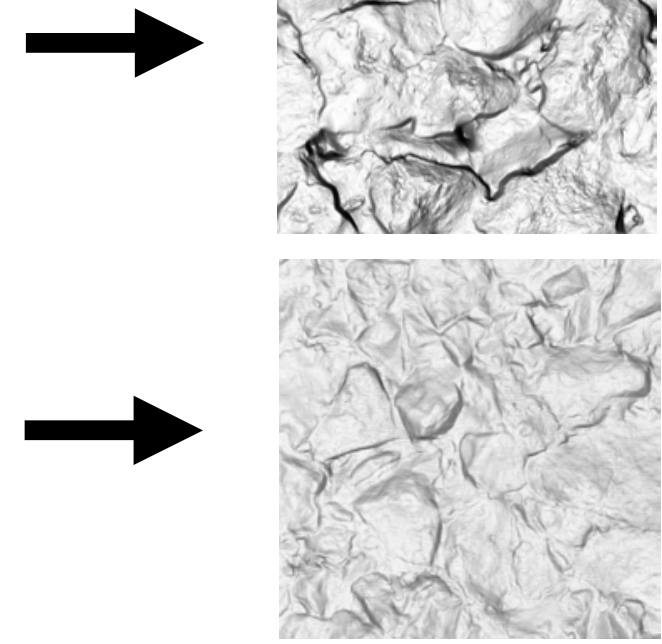

Images of the relief variation

Fig. 2: Example of road surface image and the extracted image of the relief variation.

\section{Relevance of the extracted relief and the roughness criteria}

To illustrate the relevance of the cartographies extracted by photometric stereovision, related roughness criteria were calculated and compared with friction values. Mixed criterion gradient/density was used to take into account geometric and frequential information. Fourteen road specimens were photographed; view dimensions are $9 \times 6 \mathrm{~cm}^{2}$ with 50 microns resolution. Actual surface cartographies were also measured by means of laser sensor. The mixed criterion was calculated on both "laser " and " image-extracted " cartographies. Comparison with friction values is shown in the figure 3 . It can be seen that good concordance between roughness criterion and friction values is obtained on 13 specimens.

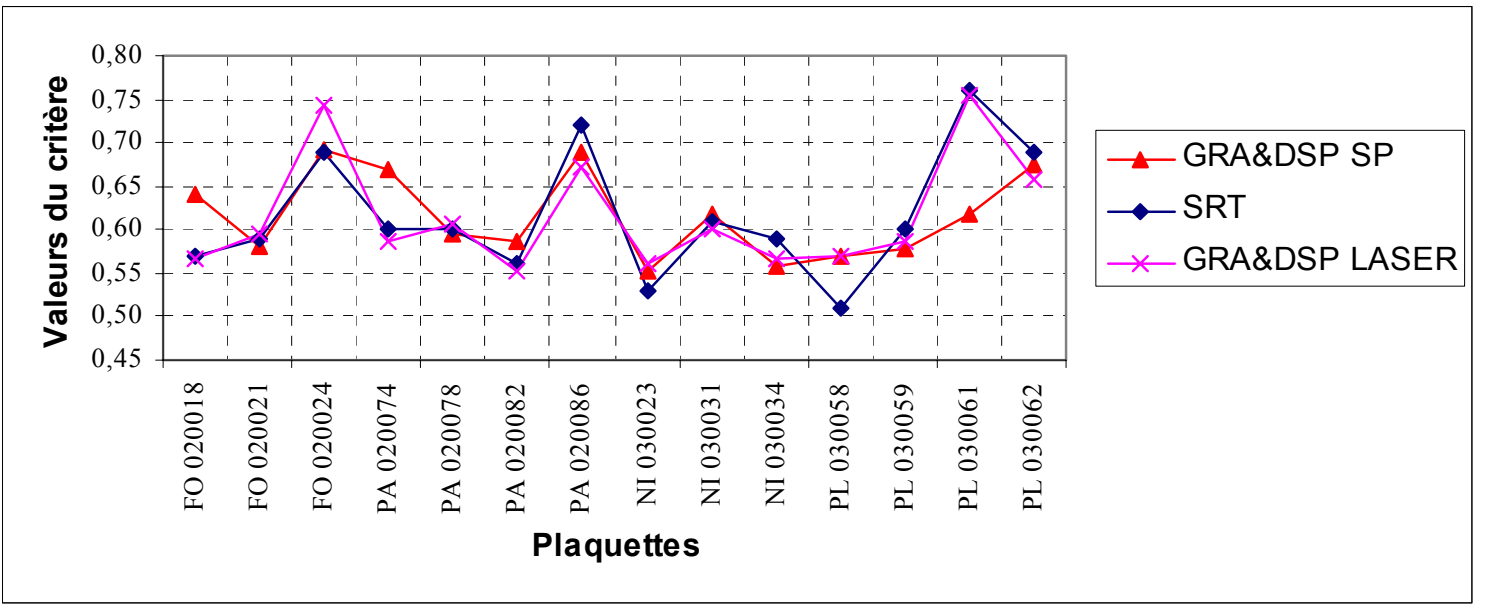

Fig. 3: Comparison between roughness criterion calculated on laser cartographies $(x)$, cartographies extracted by photometric stereovision $(\mathbf{\Delta})$ and friction coefficient SRT ( $)$. 


\section{Conclusion and perspectives}

The aim of this study is double:

- Extraction of surface cartography from image;

- Assessment of surface roughness by means of image-based descriptors.

It was shown that it is feasible to assess surface roughness by means of image analysis. Separation of relief and color information is needed prior to roughness determination. To this aim, we proposed a methodology based on photometric stereovision technique and surface photometric model. Link between relief, color and grey level can be put in equations. From surface images, it is then possible to obtain two cartographies: one related to relief and one to color. The extraction technics was applied first to grey-level images then to color images. The image resolution is 50 microns, which corresponds to road microtexture scale.

Using mixed geometric/frequential criterion, it was shown on a limited number of road specimens that the correspondence between roughness descriptors and friction values. Compared with laser cartographies, image-extracted cartographies provide equivalent results.

Further topics to be investigated in order to optimize the system performance are : system size reduction and extension of the method for on-board measurements.

\section{References}

[1] G. GRATIA, "Méthodes et Matériels de Mesure de l'Adhérence", BLPC, № 185, 1993, pp. 107-119.

[2] ISO 13473-1. "Characterization of pavement texture by use of surface profiles - Part 1: Determination of Mean Profile Depth".

[3] N. DOUIRI, M. KHOUDEIR, C. OLIVIER, "Roughness Characterization of indoor Environment", Image Analysis and Stereology Magazine, Vol. 20, $n^{\circ} 2,2001$, pp.225-229.

[4] S. PAQUIS, V. LEGEAY, H. KONIK, J. CHARRIER, "Multiresolution texture analysis applied to road surface inspection", SPIE Conference on machine vision application in industrial inspection, San Jose, USA, 1999, pp. 242-249.

[5] M. KHOUDEIR, J. BROCHARD, B. AUGEREAU, V. LEGEAY, "Discrimination of 3D textured surface: Application to the road surface wear level estimation", IAPR-International conference on Vision Interface, Montreal, 2000, pp. 174-178.

[6] M. KHOUDEIR, A. BEN SLIMANE, C. OLIVIER, "Contribution to the separation of the color information from the relief information on textured images", European Conference on Colour in Graphics, Imaging, and Vision, Poitiers, France, 2002, pp 283-286.

[7] S. MALLAT, "A theory for multiresolution signal decomposition: the wavelet representation", IEEE, PAMI, vol. 11, 1989, pp.674-693.

[8] M.OREN \& S.K.NAYAR "Generalization of Lambert's reflectance model" Computer Graphics conference SIGGRAPH'94, 1994, pp 239-246.

[9] TORRANCE, K.E. and SPARROW, E.M. "Theory for off-specular reflection from roughened surfaces" Journal of Optical Society of America, vol. 57, 1967, pp. 1105-1114.

[10] PHONG, B.T. "Illumination for computer generated pictures" Communications of the ACM, vol. 18, 1975, pp. 311-317. 